\title{
Implication of our technological species being first and early
}

\author{
Daniel P. Whitmire \\ Department of Mathematics, The University of Arkansas, Fayetteville, AR, USA e-mail: dpwhitmi@uark.edu
}

\begin{abstract}
According to the Principle of Mediocrity, a cornerstone of modern cosmology, in the absence of any evidence to the contrary, we should believe that we are a typical member of an appropriately chosen reference class. If we assume that this principle applies to the reference class of all extant technological species, then it follows that other technological species will, like us, typically find that they are both the first such species to evolve on their planet and also that they are early in their potential technological evolution. Here we argue that this suggests that the typical technological species becomes extinct soon after attaining a modern technology and that this event results in the extinction of the planet's global biosphere.

Received 18 December 2016, accepted 5 July 2017, first published online 3 August 2017
\end{abstract}

Key words: astrobiology, extraterrestrial life, intelligent life, SETI.

\section{Introduction}

In this paper, we will argue that the Principle of Mediocrity as applied to the reference class of all extant technological species leads to the inference that the typical such species is short-lived and that their demise coincides with the extinction of their global biosphere. Although we have but one datum, one is better than none and in this circumstance the Principle of Mediocrity is our best guide. The details of the evolution of life and of our technological species on the Earth may be unique. For example, on some planets technological species may have evolved much sooner than occurred on the Earth, potentially in as little as $\approx 100 \mathrm{Myr}$ (McKay 1996), and on other planets it may have taken longer. However, for the basic arguments presented here, we need to assume only that our technological species is typical in two ways: (1) that we are the first technological species to evolve on the Earth and (2) we are early in our technological evolution. Details like the particular quirks of biological and geological history that led to our technological species are not relevant for our qualitative inferences. A technological dinosaur species that evolved in $100 \mathrm{Myr}$ on a Mars-sized planet could still be first and early in their technological evolution. On the other hand, the quantitative illustrations must necessarily be based on the assumption that we are also typical in the timescales for the evolution of a technological species and the remaining planetary habitability lifetime. Assuming other (atypical) values for these timescales will result in stronger/weaker quantitative inferences.

A technological species is defined here as a biological species that has developed electronic devices and has the capacity to significantly affect their planetary environment. By this definition humans have qualified for $\approx 100$ years. The requirement of 'biological' is consistent with the Principle of Mediocrity in that if the typical technological species were nonbiological, then we would be atypical.

\section{Rapidity of the evolution of our technological species}

One-dimensional (1D) climate model calculations predicting the remaining lifetime of the Earth's biosphere have varied from $\approx 1$ to $\approx 3.3 \mathrm{Gyr}$ (Kasting et al. 1993; Rushby et al. 2013). Leconte et al. (2013) used a 3D climate model and concluded that the Earth's habitability would extend at least another $1 \mathrm{Gyr}$ into the future. More recently Wolf \& Toon (2014, 2015) used a 3D climate model and found that the Earth's climate will remain stable for another $2 \mathrm{Gyr}$, though sustained global temperatures will be too high for human survival after 1.3 Gyr (Wolf \& Toon 2015). In the following, we use this value for the future natural lifetime of the Earth's landanimal biosphere.

On the Earth, the evolution of technological life was not possible prior to the establishment of the first land animals (Tetrapods) $\approx 360$ Myr ago and probably not until the appearance of proto-primates $\approx 60 \mathrm{Myr}$ ago. By the end of the Earth's biosphere, there will have been between $\approx(360$ $\mathrm{Myr}+1.3 \mathrm{Gyr}) / 360 \mathrm{Myr} \approx 4.6$ and $\approx(60 \mathrm{Myr}+1.3 \mathrm{Gyr}) / 60$ Myr $\approx 23$ opportunities for technological species to evolve. Therefore, the evolution of our technological species occurred rapidly (the first $4.3-22 \%$ ) relative to the time period during which it could potentially have evolved. If the evolution of a technological species were improbable, we would expect to find ourselves near the end of the land-animal biosphere lifetime.

The rapid evolution of human-level intelligent life, once it was possible, might be expected as a result of convergent evolution. Flight has evolved independently four times in insects, dinosaurs, birds and bats. The basic eye has evolved independently numerous times, as has multicellularity (Grosberg \& Strathmann 2007; Parfrey \& Lahr 2013) including six times in eukaryote groups. Recently, Smith et al. (2017) found that the appendix/cecum has evolved independently 30 times in 
mammals and once evolved never disappears. High general intelligence has independently evolved at least four times, with convergent evolution in capuchins, baboons, macaques and great apes (Reader et al. 2011). Our hominin ancestors split from Neanderthals $\approx 500000$ years ago. Both species then independently (essentially) evolved brains potentially capable of creating our modern technology. This parallel convergence towards human-level intelligence may also apply to a third species, the Denisova hominin (Krause et al. 2010). The path to human-level intelligence appears to have been inevitable from at least the time of Homo Erectus. (Of course the precise path that led to our species is implausible, as is any particular evolutionary route.)

Though human-level intelligence appears to be the result of convergent evolution in terms of its inevitability, there is a significant difference between these examples of convergent evolution where the trait was independently reinvented many times and human evolution. The first technological species can (and arguably will) mitigate the repeated independent evolution of future human-level intelligent species, either intentionally if the first technological species lifetime is comparable to the planetary habitability lifetime, or unintentionally if the first technological species drastically degrades the biosphere independent of the species lifetime.

\section{Implication of the observation that we are the first technological species on the Earth}

Once possible, our technological species not only evolved rapidly but is also observed to be the first technological species on the Earth. Assuming that the Principle of Mediocrity applies to the reference class of all extant technological species, we then expect that the typical technological species will find that they too are the first such species to evolve on their planet. If our species should become extinct on timescales $\ll 1.3$ Gyr (as suggested in the section 'Implication of the observation that we are early in the potential evolution of a technological species' below) leaving the biosphere intact otherwise, then there would be numerous opportunities for additional technological species to evolve. The number of opportunities would depend on the state of the environment when our technological species became extinct. For example, if the great apes survived human extinction, we would expect only another $\approx 7 \mathrm{Myr}$ (the time since humans and apes shared a common ancestor) lapse before the evolution of the next technological species, implying as many as $\approx(7 \mathrm{Myr}+1.3 \mathrm{Gyr}) / 7 \mathrm{Myr} \approx 187$ opportunities, assuming similar future scenarios. At another extreme, if our extinction coincided with the extinction of all land life, it might take another $\approx 360 \mathrm{Myr}$ for the next technological species to evolve, yet there would still be $\approx(360 \mathrm{Myr}+1.3 \mathrm{Gyr}) /$ $360 \mathrm{Myr} \approx 4.6$ future opportunities for the evolution of other technological species. However, if our extinction coincided with the destruction of the aerobic biosphere, then the environmental reset could be as far back as $\approx 2.5 \mathrm{Gyr}$, making it unlikely that any other technological species would have time to evolve in the remaining habitable planetary lifetime. If this is the typical scenario then our (and by the Principle of
Mediocrity other technological species) seemingly privileged position as the first technological species would be expected. If the typical technological species lifetime is $\approx 1.3 \mathrm{Gyr}$, there will be time for only a single species and they would all be first. However, this is an unlikely occurrence if the Principle of Mediocrity also applies to our early technological position (see the section 'Implication of the observation that we are early in the potential evolution of a technological species' below).

The assumption of re-evolving new future technological species requires that the environment not be too drastically changed and that sufficient resources remain for the development of future technologies. Natural elemental resources like iron and aluminum are not consumed by tech species, rather their abundances are conserved since none of these elements will have left the planet (neglecting any massive space projects and even then the resources might more easily be obtained from asteroids). These resources will have been moved and processed by each technological species but they are still available. For example, a future technological species might find their iron/steel underground within the rebar of a fossil interstate or skyscraper. This form of resource may even be more easily processed and used than the original natural form. Fossil fuel resources are different, and some like coal, oil and natural gas may be significantly depleted for future technological species. There would still presumably be ample vegetation/wood available. In this situation, a newly evolved intelligent species might begin using wood as a primary energy source and then completely skip over the industrial revolution going directly to water, wind and solar power, and whatever other possibilities that might be exploited by an advanced technological species. The details of the evolution towards a new technological species will be different and it may take a longer or shorter amount of time, but the forces of convergent technological evolution are still presumably strong once a species has obtained an intelligence level comparable to that of early humans, Neanderthals, Denisova or possibly even Homo Erectus.

We can formalize the implication of being first by using Bayes' formula:

$$
P(H \mid D)=\frac{P(D \mid H) P(H)}{P(D \mid H) P(H)+P(D \mid \bar{H}) P(\bar{H})},
$$

where $H$ is the hypothesis that when a technological species becomes extinct, this event also typically results in the extinction/ reset of the biosphere; $P(H \mid D)$ is the probability of the hypothesis given the data; $D$ is the datum that we are the first technological species to evolve on the Earth; $\bar{H}=$ not- $H$ represents any other hypothesis that can explain the data; $P(D \mid H)$ and $P(D \mid \bar{H})$ are the probabilities of the data given $H$ and $\bar{H}$, respectively; $P(H)$ and $P(\bar{H})=1-P(H)$ are the prior probabilities of $H$ and $\bar{H}$ before the data are included.

We set $P(D \mid H)=1$ and $P(D \mid \bar{H})=$ statistical chance we are first $=1 / N$ where $N$ is the number of opportunities for the evolution of a technological species during the habitable lifetime of the Earth, $N=4.6-23$. We neglect two alternative hypotheses that could in principle explain the data and be included in $P(D \mid \bar{H})$. (1) The first technological species typically survives 
until the end of the planetary habitability lifetime and they prevent the independent evolution of other technological species. We argue below that the Principle of Mediocrity implies that we are typical in technological age, which strongly disfavours this hypothesis. (2) The evolution of a technological species is so improbable that it is unlikely to happen even once. If this were the case, then as noted above we would expect to find our technological species living at the end of the Earth's land-animal habitable lifetime rather than the first 4-22\%. This hypothesis also neglects the role of convergent evolution as discussed above.

Bayes' equation now reads

$$
\begin{aligned}
P(H \mid D) & =\frac{P(H)}{P(H)+(1-P(H) / N)} \\
& =\frac{1}{1-(1 / N)+1 / N P(H)} .
\end{aligned}
$$

Assuming no prior evidence, we use the uniform prior $P(H)=P(\bar{H})=1 / 2$, giving $P(H \mid D)=0.82,0.93$ and 0.96 for $N=4.6,14$ and 23, respectively. One could argue that given what we actually do know about our species' history, current and predicted environmental hazards, human nature, the Fermi paradox, and the implication of our early technological position as discussed below, $P(H)$ may be larger than $1 / 2$, corresponding to larger values of $P(H \mid D)$. On the other hand, if one is willing to believe the prior $P(H)$ is $\ll 1$, then the probability of the hypothesis $P(H \mid D)$ is small.

Under natural conditions, the Earth's biosphere has been remarkably stable over the past $500 \mathrm{Myr}$. One imaginable (if oversimplified) example of a biosphere extinction scenario is the depletion of atmospheric oxygen caused by the loss of photosynthesis in ocean phytoplankton and rain forests, possibly accelerated by rising global temperatures. Such a process may already be in progress (Boyce et al. 2010; Roxy et al. 2016). The (complete) extinction of aerobic life would reset the evolutionary clock back to the time photosynthetic oxygen first appeared in the atmosphere $\approx 2.5 \mathrm{Gyr}$ ago, leaving insufficient time for the evolution of another technological species. We (and by the Principle of Mediocrity the typical technological species) find ourselves first because there are no subsequent possibilities. In reality, the timescale for atmospheric oxygen depletion is several thousand years after the cessation of photosynthesis and humans and land animals would become extinct prior to complete oxygen depletion. Only if there were some positive feedback effect that continued to deplete atmospheric oxygen after the extinction of the technological species would there be a full $2.5 \mathrm{Gyr}$ reset, for this particular extinction scenario.

Several types of natural catastrophes, such as $\sim 10 \mathrm{~km}$ asteroid/comet impacts, nearby supernovae, $\gamma$ ray bursts and super volcanoes have been discussed and at least one of these events will probably occur in the next $100 \mathrm{Myr}$ (Sandra Faber, https://www.youtube.com/watch?v=4MBsBChHsQY). However, several of these examples have already occurred once in the last $500 \mathrm{Myr}$ and life persisted. The recovery time for the Permian-Triassic mass extinction (in which $\sim 96 \%$ of marine species and $\sim 70 \%$ of land vertebrates became extinct) is estimated to have been between 5 and $30 \mathrm{Myr}$ and that of the Cretaceous-Paleogene mass extinction (in which $\sim 75 \%$ of species became extinct) $\sim 5 \mathrm{Myr}$. The extinction event relevant to our argument here is more extreme and likely not natural.

\section{Implication of the observation that we are early in the potential evolution of a technological species}

The implication of being the first technological species on a planet implies that there are typically no subsequent technological species and that the extinction of the first generation coincides with the extinction of the global biosphere, independent of the lifetime of the first technological species. The typical first technological species could potentially survive for a very long time, including the planetary habitable lifetime. However, our own civilization is seemingly very early in the potential evolution of technology. The Principle of Mediocrity then implies that the typical technological species will observe that they too are early in the potential evolution of technology, thus arguing against the typical technological species surviving for very long timescales. As an illustration, if we assume an approximate normal distribution about a mean age $\approx$ typical age $\approx 100$ years, the standard deviation $\sigma$ of the population distribution will then lie between 0 and 100 years. Taking $\sigma \approx 100$ years, $95 \%$ of the distribution will lie at ages $<200$ years from today. This time is uncertain to at least a factor of two because the population distribution of ages cannot be strictly normal but is skewed towards older ages, since there is no absolute upper limit for the maximum age as there is for the minimum age. Consequently, we consider early technological lifetimes to mean roughly $\lesssim 500$ years.

The actual distribution of ages need not be approximately normal. However, to be consistent with the Principle of Mediocrity, the distribution should include our age of $\sim 100$ years within the middle $95 \%$ of ages. This eliminates distributions that have significant area at large ages. For example, a uniform distribution from age $=0$ to age $=1 \mathrm{Gyr}$ is unacceptable since it predicts we are among the youngest $\sim 100$ years $/ 1 \mathrm{Gyr}=10^{-7}$ technological species ages, and thus extremely atypical in violation of our assumption of the Principle of Mediocrity. At the extreme, a uniform distribution from age $=0$ to age $=4000$ years would just barely contain age $=100$ years in the middle $95 \%$. Coupled with the observation that we (and by the Principle of Mediocrity the typical technological species) are first, this implies that the typical technological species becomes extinct soon after attaining technological status and that this event results in the extinction of the global biosphere.

The approximate normal distribution illustration that the lifetime of the typical technological species is $\lesssim 500$ years is subject to a sampling bias in that the question cannot be asked until a modern high-tech species exists. If the questions were asked 1000 years from now, the predicted lifetime would be $<5000$ years. If asked in one million years, the predicted lifetime would be less than five million years, and so on. Though this is true, it is only relevant if the typical technological species 
survives that far into its future. Using the above estimate assuming an approximate normal distribution (and neglecting outliers), the probability of survival to times $>500$ years is negligible. At the other extreme, how soon could these questions have been asked? The fact that we are the first technological species to evolve on the Earth could not have been known too much earlier than the present time, in coincidence with us qualifying as a technological species. The modern version of the Principle of Mediocrity and the Anthropic Principle (see below) probably could not have been formulated too much earlier than they were. So the questions addressed here could have been asked somewhat earlier, but they are guaranteed to be asked relatively soon after a modern technology is attained. Therefore, other early technological species will, like us, infer that the typical such species will be short-lived and likely destructive of their global biosphere. This inference will be made independent of whether or not it is correct for that specific technological species. In our application of the Principle of Mediocrity, the reference class is all extant technological species.

Our Principle of Mediocrity inference based on us being technologically early is not the same logic as the so-called Doomsday Argument (Carter \& McCrea 1983; Gott 1993; Leslie 1996; Bostrom 2002), which itself is an unjustified extension of the German Tank Problem. The most common version of the Doomsday Argument holds that your birth order rank ( $\approx 100$ billion) is expected (i.e. you are typical) if the total number of humans that will ever have lived is $\approx 2 \times 100$ billion, but is highly improbable if the total number is much larger, say $10^{18}$. The implication is that humans will only survive for the time it takes the present population to roughly double. The flaw in this argument is the fundamental assumption that you are a random observer selected from the set of all humans that will ever have existed.

In World War II, the allies estimated the total number of Panzer 5 German tanks on the basis of serial numbers obtained from captured or destroyed tanks (see en.wikipedia.org/wiki/ German_tank_ problem for the historical background and analysis). The statistical estimate was remarkably accurate, much better than the conventional intelligence estimates, when compared with the post-war documentation. The analysis can be done with as few as one serial number from one tank. The frequentist prediction estimate in this case is that the total number of tanks is about twice the serial number. This is also intuitive. For example, given a single serial number $=4$, we would not likely bet on a total number of $\approx 10000$ tanks. A better bet would be for a total number $\approx 8$, assuming 4 is a random selection from all the existing tanks.

The Doomsday Argument and the German Tank problem are similar up to a point. However, the German Tank problem equivalent of the Doomsday Argument is to estimate the total number of tanks that will ever exist. This is not the same problem at all and could not have been done from the existing serial numbers during World War II. We could do this if we had a random selection of serial numbers from all tanks that will have ever existed. The equivalent Doomsday problem is the same. We are not selected from a random sample of all humans that have or ever will have existed since unrealized future humans are not available for selection. My Dell laptop computer is not a random computer selected from all computers that have or ever will have existed. It can only exist now. Likewise, you are a unique person with your own genetic blueprint and memories and you can only exist now. You cannot be Cleopatra or Buck Rogers, just like my laptop computer with its own electronics and memory cannot be a 1960s main frame or a 28 th century computer implant. It is not a computer that has been randomly selected from the ensemble of all computers that will ever have existed. A chosen tank serial number or human birth order cannot be typical of all past and future tanks or humans since unrealized tanks and humans are not available for the random selection process. On the other hand, the Principle of Mediocrity argument does not depend on unrealized future technological species. Typicality need only be defined in terms of existing technological species. Nothing is assumed or implied regarding the frequency of technological species in the universe other than that some statistically significant number exist, for example, $\geq 100$. Given that there are probably over $\sim 10^{22}$ planets in the known universe, this assumption seems safe.

\section{The Principle of Mediocrity}

The heuristic Principle of Mediocrity (also known as the Copernican Principle and Typicality Principle) is the precept that, in the absence of any evidence to the contrary, we should assume that we are typical members of an appropriately chosen reference class (Shklovskii \& Sagan 1966a; Vilenkin 2011). The Principle of Mediocrity has become an important consideration in modern inflationary multiverse cosmology where it is used to justify the local testing of global predictions (Garriga \& Vilenkin 2008; Vilenkin 2011). Not being merely philosophical, the Principle of Mediocrity can be employed as a winning betting strategy (Vilenkin 2011). The idea can be extended beyond ourselves as individuals, or our species, or our civilization, and be applied to other objects, processes or attributes as well.

The Principle of Mediocrity is sometimes considered controversial because of the potential vagueness of the definition of the reference class. The Principle is guaranteed to be tautologically correct if we define the reference class sufficiently narrow. For example, a 1999 Acura automobile is guaranteed to be a typical member of the reference class of all identical models of 1999 Acura automobiles still in use. In the case of our technological species, we are guaranteed to be a typical member of the reference class of all technological species identical to ours. However, the Principle of Mediocrity can still be useful when we relax such (by definition) specificity. If the sole information we have about automobiles is that obtained from an examination of a single 1999 Acura and we wish to predict properties of other automobiles in the world, we would have little recourse but to appeal to the Principle of Mediocrity. The primary reference class is automobiles, which we can define as four-wheel transportation vehicles for humans. We now assume that the 1999 Acura is also typical in secondary 
properties, such as size, age, horsepower, maximum speed, diameter of the wheels, etc. We can define typical as being within two standard deviations of the population mean of the attribute, for example. In all or most of the various possible attributes, this 1999 Acura will be found to be typical, and so in this example, the Principle of Mediocrity is confirmed. Note that we should expect a small fraction $(\approx 5 \%)$ of secondary attributes to be atypical. In the primary reference class of humans on the Earth you are probably typical (within two standard deviations) in most of your secondary attributes, such as height, weight, walking speed, age, body temperature, etc.

An example of a counter argument to the Principle of Mediocrity that has been given is, within the reference class of all objects of mass $10-100 \mathrm{~kg}$, we humans are not typical. We are typical of the primary reference class with masses lying within this range. In terms of secondary attributes like elemental abundances we are not typical of this reference class. However, this example was chosen specifically because it was known a priori not to satisfy the Principle of Mediocrity in terms of secondary attributes. It required prior knowledge of humans and rocks, so there was prima facie contrary evidence to applying the Principle of Mediocrity. The Principle is only applied as the best guide in the absence of any mitigating evidence.

For some applications, like the ones mentioned above, there is prior knowledge that either supports or does not support the application of the Principle of Mediocrity. An example of an unbiased test of the Principle that could have been done is the discovery of pulsars. When the first radio pulsar was discovered in 1967, it was a unique and enigmatic phenomenon. Original hypotheses included alien signals, binary compact objects, oscillating white dwarfs and neutron stars. Given that there was no prior knowledge, the Principle of Mediocrity could have been invoked for this single event. The period of this first discovered pulsar PSR B1919 + 21 was $1.3 \mathrm{~s}$, and so we could have predicted that this period is typical, that is, once the period population distribution was known, this pulsar's period would be found to lie within two standard deviations of the population mean. Subsequently, large numbers of radio pulsars were discovered and their mean period was found to be $\approx 0.8 \mathrm{~s}$. The first pulsar period of $1.3 \mathrm{~s}$ was comfortably within two standard deviations $\approx \pm 1 \mathrm{~s}$.

The Principle of Mediocrity is not at all the same as the Anthropic Principle, as is sometimes implied, and in fact they are in a sense antithetical, though complementary. The noncontroversial Anthropic Principle (sometimes called the Weak Anthropic Principle) is a selection effect that says when interpreting observations, we should consider the filter of our own existence. In applying the Principle of Mediocrity, we should take into account any anthropic selection effects that may be relevant to our reference class. For example, the Sun is more massive and luminous than the typical star $(\approx 95$ percentile). If we condition on life, the distribution of stars makes the Sun more typical, and if we condition on technological life, the Sun is virtually at the peak of the mass distribution (Whitmire \& Matese 2009). Similarly, the Earth is not a typical planet, but if we condition on life, it is more typical (rocky planet with water in the Habitable Zone), and if we condition on technological life, it is likely to be quite typical (oxygen atmosphere, continents, plate tectonics, possibly a large moon). Likewise our Solar System may not be typical of other planetary systems (e.g. in the absence of super-Earths) but may be typical of planetary systems that contain a planet with a technological species. If you pick up a rock, it will not likely be typical of all rocks on the Earth since the great majority of rocks will be smaller. On the other hand, the chosen rock may well be typical of rocks picked up by humans.

Our interest here is in applying the Principle of Mediocrity to the reference class of all extant technological species in the universe. We previously defined technological species as those biological species that have developed the ability to affect the global environment and utilize electronic devices. Since we have no prior knowledge of other technological species at this point, the Principle of Mediocrity is our best guide. The Fermi paradox has many proposed explanations, so it does not represent prior knowledge, or contrary evidence, that is, it does not imply that we are atypical of technological species. There is a spectrum of secondary characteristics within the reference class of extant technological species, but we focused here on the two most salient ones. If we are typical of this reference class, then other members would, like us, observe that their technological species is the first to evolve on their planet and also that they are early in their potential technological evolution. Our inferences are based on this application of the Principle of Mediocrity.

\section{Implication for $L$ in the Drake equation}

Our application of the Principle of Mediocrity implies a very small value for the typical lifetime of technological species, but this does not necessarily mean that the average lifetime $L$ in the Drake equation is correspondingly small. If there is even a small fraction of very long-lived technological species, then the mean lifetime $L$ will still be significant (Shklovskii \& Sagan 1966b). For example, if $99 \%$ of technological species have lifetimes of 100 years and $1 \%$ have lifetimes of $1 \mathrm{Gyr}$, the average lifetime is $10^{7}$ years, corresponding to $\sim 10^{6}$ extant technological species in our Galaxy, using common estimates for the various other factors. Though not warranted here, this situation could be better modelled using the heavy tailed Levy distribution. This distribution has the desired properties of positive values of the random variable (age), a finite median, mode and scale parameter (analogous to a standard deviation) and mean $=$ infinity (allowing for the possibility that the mean is $>$ median and scale parameter).

Although we do not use the Fermi Paradox or SETI project results in our arguments, we note that the implied short lifetime of the typical technological species is consistent with both.

\section{Conclusion}

Our inferences regarding the fate of the typical technological species are based on two observations and essentially one assumption. The observations are that our technological species is (1) the first such species to evolve on the Earth and (2) early 
in its potential technological evolution. The assumption is that the Principle of Mediocrity applies to the reference class of all extant technological species. Given this assumption, the suggested inference is that the typical technological species has a short lifetime and that their extinction coincides with the extinction of their planetary biosphere.

\section{Acknowledgements}

The author is indebted to an anonymous referee for a thoughtful and thorough review and suggestions that significantly improved the manuscript. The author thanks Professor Jason K. Whitmire for comments on an earlier version of the paper.

\section{References}

Bostrom, N. (2002). Anthropic Bias: Observational Selection Effects in Science and Philosophy, ch. 6. Routledge, NY.

Boyce, D.G., Lewis, M.R. \& Worm, B. (2010). Global phytoplankton decline over the past century. Nature 466, 591-596.

Carter, B. \& McCrea, W.H. (1983). The Anthropic Principle and its implications for biological evolution. Philos. Trans. R. Soc. Lond. A310 (1512), 347-363.

Garriga, J. \& Vilenkin, A. (2008). Prediction and explanation in the multiverse. Phys. Rev. D 77, 043526, arXiv:0711.2559v3.

Gott, R. (1993). Implications of the Copernican Principle for our future prospects. Nature 363, 315-319.

Grosberg, R.K. \& Strathmann, R.R. (2007). The evolution of multicellularity: a minor major transition? Annu. Rev. Ecol. Evol. Syst. 38, 621-624.

Kasting, J.F., Whitmire, D.P. \& Reynolds, R.T. (1993). Habitable zones around main sequence stars. Icarus 101, 108-128.
Krause, J., Fu, Q., Good, J.M., Viola, B., Shunkov, M.V., Derevianko, A.P. \& Pääbo, S. (2010). The complete mitochondrial DNA genome of an unknown hominin from southern Siberia. Nature 464(7290), 894-897.

Leconte, J., Forget, F., Charnay, B., Wordsworth, B. \& Pottier, A. (2013). Increased insolation threshold for runaway greenhouse processes on Earth-like planets. Nature 504, 268-271.

Leslie, J. (1996). The End of the World: The Science and Ethics of Human Extinction. Routledge, London, pp. 187-236.

McKay, C. (1996) Circumstellar Habitable Zones. Travis House Pub., Menlo Park, CA.

Parfrey, L.W. \& Lahr, D.J.G. (2013). Multicellularity arose several times in the evolution of eukaryotes. Bioessays 35(4), 339-347.

Reader, S.M., Hager, Y. \& Laland, K.N. (2011). The evolution of primate general and cultural intelligence. Philos. Trans. R. Soc. Lond. B Biol. Sci. 366(1567), 1017-1027.

Roxy, M.K., Modi, A., Murtugudde, R., Valsala, V., Panickal, S., Kumar, S. P., Ravichandran, M., Vichi, M. \& Levy, M. (2016). A reduction in marine primary productivity driven by rapid warming over the tropical Indian Ocean. Geophys. Res. Lett. 43, 826-833.

Rushby, A.J., Mark, W.C., Osborn, H. \& Watson, J. (2013). Habitable zone lifetimes of exoplanets around main sequence stars. Astrobiology 13(9), 833-849.

Shklovskii, I.S. \& Sagan, C. (1966a). Intelligent Life in the Universe. Dell Pub. Co. Inc., New York, pp. 356-361.

Shklovskii, I.S. \& Sagan, C. (1966b). Intelligent Life in the Universe. Dell Pub. Co. Inc., New York, pp. 413.

Smith, H.F., Parker, W., Kotzé, S.H. \& Laurin, M. (2017). Morphological evolution of the mammalian cecum and cecal appendix. C. R. Palevol 16 (1), 39-57.

Vilenkin, A. (2011). The principle of mediocrity. Astron. Geophys. 52(5), 5.33-5.36. http://cosmos2.phy.tufts.edu/Vilenkin.Whitrow.pdf, http:// arxiv.org/abs/1108.4990.

Whitmire, D.P. \& Matese, J.J. (2009). The distribution of stars most likely to harbour intelligent life. Astrobiology 9(7), 617-621.

Wolf, E.T. \& Toon, O.B. (2014). Delayed onset of runaway and moist greenhouse climates for Earth. Geophys. Res. Lett. 41, 167-142.

Wolf, E.T. \& Toon, O.B. (2015). The evolution of habitable climates under the brightening Sun. J. Geophys. Res. Atmos. 120, 5775-5794. 